\title{
Lack of Association between LCT_rs 140433552*CA>del Indel Polymorphism and Lactose Intolerance in a Southern Brazilian Population
}

\author{
Luana Caroline Oliveira ${ }^{a, b} \quad$ Andrey Lucas Dias Barros ${ }^{b} \quad$ Angelica Beate Winter Boldt $^{a}$ b \\ Gabriel Adelman Cipolla ${ }^{a, b}$ \\ a Postgraduate Program in Genetics, Department of Genetics, Federal University of Paraná (UFPR), Curitiba, Brazil; ${ }^{\mathrm{b}}$ Laboratory \\ of Human Molecular Genetics, Department of Genetics, Federal University of Paraná (UFPR), Curitiba, Brazil
}

\section{Keywords}

rs 140433552*CA > del $\cdot$ Indel $\cdot 3^{\prime}$ untranslated region $\cdot$ LCT . Haplotypes · Lactose intolerance $\cdot$ Genetic susceptibility

\begin{abstract}
Background/Aims: Polymorphisms in the enhancer of the lactase gene $(L C T)$ are strongly associated with lactase persistence, but not always predictive of the phenotype. We investigated a possible association between the regulatory rs $140433552^{*}$ CA $>$ del variant of LCT and lactose intolerance (LI).Methods: We genotyped 122 individuals for rs140433552 and rs4988235 $\left(-13910^{*} \mathrm{C}>T\right)$. Results: Associations of rs $140433552^{*} \mathrm{CA}>$ del with $\mathrm{LI}$ depend on $-13910^{*} \mathrm{C}>\mathrm{T}$. Homozygous individuals for the $C$-CA haplotype, as well as $C-C A+/ C$ individuals, seem more likely to manifest LI (OR $3.33[95 \% \mathrm{Cl}$ 1.32-8.35], $p=0.011$, and OR 3.93 [95\% Cl 1.61-9.61], $p=$ 0.003 , respectively), while homozygous individuals for the T-CA haplotype seem more likely to be lactose tolerant (OR 0.04 [95\% Cl 0.002-0.70], $p=8 \times 10^{-4}$ ). Conclusions: rs $140433552^{*}$ CA $>$ del is not independently associated with LI.

(c) 2020 The Author(s)

Published by S. Karger AG, Basel
\end{abstract}

\section{Introduction}

Lactase non-persistence or primary hypolactasia is the inability to hydrolyze lactose, an abundant disaccharide in milk, resulting in lactose intolerance (LI). This process is genetically programmed, and in most mammals, at weaning time, physiological downregulation of the lactase gene $(L C T)$ occurs, resulting in low lactase activity throughout adult life [1]. An evolutionary adaptation allowed certain human groups with an ancient history of milk and dairy production and consumption - such as Northern European populations, as well as nomads of Africa and of the Middle East - to digest lactose even in adulthood [1-3], a phenotype named lactase persistence (LP) or lactose tolerance (LT).

Several single nucleotide polymorphisms in a transcriptional enhancer region of $L C T$ have been associated with LP. In Northern Europeans, the $-13910^{*} T$ and $-22018^{*} G$ alleles located $\sim 14$ and $\sim 22 \mathrm{~kb}$ upstream of $L C T$, within introns 13 and 9 of minichromosome maintenance complex component 6 gene (MCM6), respectively, are significantly associated with LP [4]. Functional studies have indicated that the $-13910^{*} T$ allele is the key variant regulating the maintenance of $L C T$ expression in

Gabriel Adelman Cipolla

Laboratory of Human Molecular Genetics, Department of Genetics

Federal University of Paraná, Av. Coronel Francisco Heráclito dos Santos, 100

Curitiba, PR 81530-900 (Brazil)

gabriel.cipolla@ufpr.br 
Europeans [5]. However, in some cases, $-13910^{*} C>T$ genotypes are not strictly predictive of the phenotype. A physiological change in lactase gene expression occurs in the context of a stable DNA sequence, suggesting the presence of other dynamic mediators of LCT expression regulation [6].

The $3^{\prime}$ untranslated region ( $\left.3^{\prime} \mathrm{UTR}\right)$ is generally considered to be an important sequence for posttranscriptional regulation of gene expression by harboring microRNA (miRNA) targeting sites [7]. In turn, miRNA are small, noncoding RNA molecules that bind to the $3^{\prime} \mathrm{UTR}$ to direct messenger RNA degradation or translational inhibition, therefore acting as negative regulators of gene expression [8]. Hence, genetic polymorphisms in the $3^{\prime}$ UTR of genes may affect gene expression by creating new or disturbing original miRNA binding sites [9]. The rs 140433552 is a 2-bp indel located in the $3^{\prime}$ UTR of LCT. According to the software PolymiRTS [10], this indel may disrupt the binding of two miRNAs (hsa-miR-4731-3p and hsa-miR-4801) in transcripts harboring the deletion. In functional terms, this deletion could contribute to a higher expression of LCT.

In the present study, we aimed to investigate, for the first time, an association between rs140433552 polymorphism and LI in a population of European descendants from Southern Brazil.

\section{Subjects and Methods}

\section{Research Participants}

For this study, a total of 43 lactose-intolerant individuals (LI group; 32 women/11 men, aged 18-71 years) and 79 lactose-tolerant individuals (LT group; 49 women/30 men, aged 14-74 years) were included. Data for both groups were collected through interviews and medical records. Inclusion criteria were: (1) written informed consent; and (2) European ancestry determined by physical characteristics, in agreement with self-reported ancestry. Specifically for LI, we only included individuals with gastrointestinal symptoms and a negative result for the LT test. This test measures the blood glucose levels immediately before and 2-6 times, in 15to 30-min intervals, after oral administration of lactose, with a maximum rise of $20 \mathrm{mg} / \mathrm{dL}$ at any timepoint within $2 \mathrm{~h}$ indicating LI (i.e., a negative result) [11]. Exclusion criteria were: (1) consanguinity with any individual from either group; (2) disagreement between inferred and self-reported ancestries; and (3), specifically for LT, a familial history of LI.

\section{LCT Genotyping}

Blood was collected in tubes containing the anticoagulant EDTA, and genomic DNA was extracted from whole blood according to the Wizard ${ }^{\circledR}$ Genomic DNA Purification kit protocol (Promega, Madison, WI, USA). The $r s 140433552^{*} C A>d e l$ genotype was identified using PCR-SSP, with allele-specific forward primers. For the amplification of a 212-bp fragment, we used the following primers: forward primers $r s 140433552^{*} \mathrm{CA}$ (5'-CTGCTGTTTTTATTTTCTGGAAAACAC- $\left.3^{\prime}\right)$ and $r s 140433552^{*} \mathrm{del}$ ( $5^{\prime}$-CTGCTGTTTTTATTTTCTGGAAAACAA- $\left.3^{\prime}\right)$, and reverse primer 140433552_rev(5'-GGTCTAGTGGGTCTGTGAACC- $\left.3^{\prime}\right)$. All PCR co-amplified a fragment of 431 bp of human growth hormone gene $(H G H)$ as an overall control for amplification (forward primer $5^{\prime}$-TGCCTTCCCAACCATTCCCTTA- $3^{\prime}$ and reverse primer $5^{\prime}$-CCACTCACGGATTTCTGTTGTGTTTC-3').

All reactions were carried out in a final volume of $8 \mu \mathrm{L}$, containing $20 \mathrm{ng}$ of genomic DNA, $0.15 \mathrm{~mm}$ of each $H G H$ primer, $0.3 \mathrm{~mm}$ of each rs140433552 primer, $0.2 \mathrm{mM}$ of each dNTP and $1 \times$ Coral Buffer (Invitrogen Life Technologies, Carlsbad, CA, USA). Thermal cycling conditions began with $94^{\circ} \mathrm{C}$ for $2 \mathrm{~min}$, followed by a touchdown strategy $\left(7 \mathrm{cycles}\right.$ at $65^{\circ} \mathrm{C}, 15$ at $63^{\circ} \mathrm{C}$, and 7 at $61^{\circ} \mathrm{C}$ ) on an Eppendorf Mastercycler EP Gradient 96 well Thermal Cycler (Hamburg, Germany). Amplified fragments were run on $1.5 \%$ agarose gel, and stained with UniSafe Dye (Uniscience, São Paulo, Brazil). To better characterize the sample, we also performed genotyping for $r s 4988235^{*} C>T\left(-13910^{*} C>T\right)$. This variation is strongly associated with LP in European populations. The sequence-specific primers and PCR-SSP conditions for this single nucleotide polymorphism were based on Boschmann et al. [12].

\section{Statistical and Bioinformatic Analysis}

We obtained allelic, genotypic, and haplotypic frequencies by direct counting, and tested the hypothesis of Hardy-Weinberg equilibrium by the exact test of Guo and Thompson. We reconstructed extended haplotypes based on linkage disequilibrium with HaploView 4.2. We further compared the distribution of alleles, genotypes, and haplotypes between the LI and the LT group using Fisher's exact test in the online software VassarStats. The odds ratio (OR) was calculated with a $95 \%$ CI. $p$ values $<0.05$ were considered significant.

\section{Results}

A total of 122 individuals were genotyped for rs $140433552^{*} C A>d e l$, and for $r s 4988235^{*} C>T$, the main LT-associated variant. The genotypic distributions in both groups were in agreement with those predicted under Hardy-Weinberg equilibrium. The allelic and genotypic frequencies of $r s 140433552 * C A>d e l$ in our LI group were similar to those in the Iberian and British populations from the 1000 Genomes Project [13]. We did not find evidence of any association between LI and rs140433552 alleles or genotypes (Table 1).

According to the prediction algorithm PolymiRTS, the 2-bp deletion in the context of rs140433552 disrupts the binding site of two miRNAs. This could contribute to and be associated with continued lactase enzyme expression in individuals with the $C / C$ genotype who are lactose tolerant. In order to test if such 2-bp deletion could account in part for LT, we compared allelic and genotypic fre- 
Table 1. Association of polymorphisms with LI

\begin{tabular}{|c|c|c|c|c|c|}
\hline & $\begin{array}{l}\text { LI group } \\
(n=43), n(\%)\end{array}$ & $\begin{array}{l}\text { LT group } \\
(n=79), n(\%)\end{array}$ & OR & $95 \% \mathrm{CI}$ & $p$ value \\
\hline \multicolumn{6}{|c|}{ rs $140433552^{\star} \mathrm{CA}>\mathrm{del}$} \\
\hline$C A$ & $60(69.8)$ & $125(79.1)$ & 0.60 & $0.33-1.10$ & 0.118 \\
\hline$C A / C A$ & $20(46.5)$ & $47(59.5)$ & 0.59 & $0.28-1.25$ & 0.186 \\
\hline CA/del & $20(46.5)$ & $31(39.2)$ & 1.34 & $0.63-2.85$ & 0.449 \\
\hline $\mathrm{del} / \mathrm{del}$ & $3(7.0)$ & $1(1.3)$ & 5.85 & $0.58-58.06$ & 0.125 \\
\hline$C A+$ & $40(93.0)$ & $78(98.7)$ & 0.17 & $0.01-1.69$ & 0.125 \\
\hline del+ & $23(53.5)$ & $32(40.5)$ & 1.69 & $0.79-3.57$ & 0.186 \\
\hline \multicolumn{6}{|c|}{$r s 4988235^{\star} C>T$} \\
\hline C & $73(84.9)$ & $87(55.1)$ & 4.58 & $2.34-8.93$ & $2 \times 10^{-6}$ \\
\hline CC & $30(69.8)$ & $25(31.7)$ & 4.98 & $2.22-11.15$ & $6 \times 10^{-5}$ \\
\hline$C T$ & $13(30.2)$ & $37(46.8)$ & 0.49 & $0.22-1.08$ & 0.086 \\
\hline$T T$ & $0(0)$ & $17(21.5)$ & 0.04 & $0.002-0.70$ & $8 \times 10^{-4}$ \\
\hline$C+$ & $43(100)$ & $62(78.5)$ & 24.36 & $1.42-415.98$ & $8 \times 10^{-4}$ \\
\hline \multirow[t]{2}{*}{$T+$} & $13(30.2)$ & $54(68.4)$ & 0.20 & $0.08-0.44$ & $6 \times 10^{-5}$ \\
\hline & $\begin{array}{l}\text { LI group } \\
(n=30), n(\%)\end{array}$ & $\begin{array}{l}\text { LT group } \\
(n=25), n(\%)\end{array}$ & OR & $95 \% \mathrm{CI}$ & $p$ value \\
\hline \multicolumn{6}{|c|}{$r s 4988235^{\star} \mathrm{C} / \mathrm{C}$} \\
\hline$C A$ & $41(68.3)$ & $34(68.0)$ & 1.01 & $0.45-2.27$ & 1 \\
\hline$C A / C A$ & $14(46.7)$ & $10(40.0)$ & 1.17 & $0.41-3.36$ & 0.795 \\
\hline CA/del & $13(43.3)$ & $14(56.0)$ & 0.42 & $0.20-1.75$ & 0.421 \\
\hline del/del & $3(10.0)$ & $1(4.0)$ & 2.66 & $0.25-27.38$ & 0.617 \\
\hline$C A+$ & $27(90.0)$ & $24(96.0)$ & 1.05 & $0.43-2.57$ & 1 \\
\hline del+ & $19(31.7)$ & $16(32.0)$ & 0.84 & $0.29-3.36$ & 0.795 \\
\hline
\end{tabular}

Bold values indicate statistical significance at $p<0.05$. All genotypic distributions are in agreement with the Hardy-Weinberg equilibrium. LI, lactose intolerance; LT, lactose tolerance; $n$, number of individuals; OR, odds ratio by binary logistic regression; $\mathrm{CI}$, confidence interval.

Table 2. Association of haplotypes with LI

\begin{tabular}{lccccc}
\hline Haplotypes & $\begin{array}{l}\text { LI group } \\
(n=43), n(\%)\end{array}$ & $\begin{array}{l}\text { LT group } \\
(n=79), n(\%)\end{array}$ & OR & $95 \%$ CI & $p$ value \\
\hline C-CA/C-CA & $14(32.6)$ & $10(12.7)$ & 3.33 & $1.32-8.35$ & $\mathbf{0 . 0 1 1}$ \\
C-del/C-CA & $13(30.2)$ & $14(17.7)$ & 2.01 & $0.84-4.80$ & 0.169 \\
C-del/C-del & $3(7.0)$ & $1(1.3)$ & 5.85 & $0.58-58.06$ & 0.125 \\
C-CA/T-CA & $6(13.9)$ & $20(25.3)$ & 0.47 & $0.17-1.30$ & 0.170 \\
C-del/T-CA & $7(16.3)$ & $17(21.5)$ & 0.70 & $0.26-1.87$ & 0.634 \\
T-CA/T-CA & $0(0.0)$ & $17(21.5)$ & 0.04 & $0.002-0.70$ & $\mathbf{8} \times \mathbf{1 0}^{-4}$ \\
C-CA+/C & $27(72.9)$ & $24(40.7)$ & 3.93 & $1.61-9.61$ & $\mathbf{0 . 0 0 3}$ \\
C-del+/C & $16(44.4)$ & $15(24.2)$ & 2.50 & $1.04-6.02$ & $\mathbf{0 . 0 4 5}$ \\
\hline
\end{tabular}

Bold values indicate statistical significance at $p<0.05$. Analyses were performed using the sum of all other haplotypes as a reference. Haplotypes represent two investigated variants (rs4988235 and rs140433552). LI, lactose intolerance; LT, lactose tolerance; $n$, number of individuals; OR, odds ratio; CI, confidence interval.

3'UTR Indel of the Lactase Gene and Lactose Intolerance
Lifestyle Genomics 2020;13:129-133 DOI: $10.1159 / 000508509$ 
quencies only among individuals with the $r s 4988235^{*} \mathrm{C} / \mathrm{C}$ genotype. Thirty lactose-intolerant individuals and 25 lactose-tolerant individuals with the $r s 4988235^{*} \mathrm{C} / \mathrm{C}$ genotype were included. However, we did not observe any statistically significant differences between the LI and the LT groups for the frequencies of $r s 140433552 *$ del homozygotes or carriers (OR 2.66 [95\% CI 0.25-27.38], $p=$ 0.94 , and OR 0.66 [95\% CI 0.38-2.31], $p=1$, respectively; Table 1).

We also tested for the association of rs4988235 and rs140433552 haplotypes with LI (Table 2). There was an association when evaluating both investigated polymorphisms. Homozygous individuals for the $C$ - $C A$ haplotype and $C-C A+/ C$ individuals are more likely to be lactose intolerant (OR 3.33 [95\% CI 1.32-8.35], $p=0.011$, and OR 3.93 [95\% CI 1.61-9.61], $p=0.003$, respectively), while homozygous individuals for the T-CA haplotype are more likely to be lactose tolerant (OR 0.04 [95\% CI 0.002-0.70], $p=8 \times 10^{-4}$ ).

\section{Discussion}

It is well accepted that lactase gene expression is primarily regulated at the transcriptional level [6], and that rs4988235 is associated with LP in people of European ancestry [3]. However, in some cases, these genotypes are not truly predictive of the phenotype. In the present study, we hypothesized that $r s 140433552^{*} C A>d e l$, located in the $3^{\prime}$ UTR of $L C T$, could be associated with LI, given its potential to disrupt a miRNA targeting site and, therefore, interfere with enzyme levels.

Genetic polymorphisms in the $3^{\prime} \mathrm{UTR}$ of genes have been shown to significantly affect gene expression, creating new or disrupting original miRNA binding sites. These polymorphisms may mimic loss-of-function mutations, leading to loss of miRNA function and, consequently, affectinggeneexpression [9]. Thers $140433552^{*} \mathrm{del}$ allele has been predicted to disrupt the binding of two miRNAs (hsa-miR-4731-3p and hsa-miR-4801) at their original binding sites.

However, no evidence of association between LI and alleles or genotypes of rs140433552 was observed. Likewise, we found no association when analyzing whether rs $140433552 * \mathrm{del}$ is associated with LT among individuals with the $r s 4988235^{*} C / C$ genotype. Nonetheless, homozygous individuals for the $C$ - $C A$ haplotype, as well as $C$ $C A+/ C$ individuals, seem more likely to manifest the LI trait. Considering that homozygous individuals for the $T$-CA haplotype seem more prone to manifest LT, it is reasonable to suggest that rs140433552 does not play a major role in the trait, since the $C A$ allele is present in both haplotypes of contrasting effects. Analyses evaluating the relationship between these haplotypes and enzyme levels could deliver another degree of evidence to our findings.

Our study is the first to address rs140433552 in the context of LI. This $3^{\prime} \mathrm{UTR}$ indel polymorphism of $L C T$ is not independently associated with this phenotype in our sample. However, studies with larger groups and individuals of different ethnicities are necessary to better explore the role of $r s 140433552^{*} \mathrm{CA}>$ del in LI, if any. Moreover, approaches exploring the functional role of this polymorphism in LCT expression could help understand its physiological relevance in lactase non-persistence.

\section{Acknowledgments}

We warmly thank the participants who volunteered for this study, the Associação de Celíacos do Paraná (ACELPAR) for supporting our work, and the members of the Laboratory of Human Molecular Genetics of the UFPR.

\section{Statement of Ethics}

This study followed all the guidelines of the National Council for Ethics in Research (Conselho Nacional de Ética em Pesquisa CONEP, Brazil) for research with humans. Every participant gave written informed consent in agreement with the Declaration of Helsinki. The Ethics Committee of the Health Sciences Sector of the Federal University of Paraná (Comitê de Ética em Pesquisa do Setor de Ciências da Saúde da UFPR - CEP SCS-UFPR, Curitiba, Brazil) approved this study (protocols 2.247.952 - CAAE 55297916.6.0000.0102and2.204.113-CAAE54385616.2.0000.0102).

\section{Conflict of Interest Statement}

All authors declare that this research has been conducted in the absence of any conflict of interest.

\section{Funding Sources}

This work was supported by: Fundação Araucária-PR; SESAPR; MS-Decit (CP 01/16 - PPSUS); Conselho Nacional de Desenvolvimento Científico e Tecnológico (CNPq); and Coordenação de Aperfeiçoamento de Pessoal de Nível Superior (CAPES/PROAP - Finance Code 001). L.C.O. received a PhD scholarship (CAPES program 40001016006P1) and G.A.C. received a postdoctoral scholarship (CAPES protocol 88882.306040/2018-01) both from CAPES. A.B.W.B. currently receives a research productivity scholarship from CNPq (CNPq protocol 314288/2018-0). 


\section{Author Contributions}

All authors contributed to the conception of this study; L.C.O. and G.A.C. designed the study; L.C.O. and A.B.W.B. contacted the participants to take part in this study; A.B.W.B. pro- vided the funds through research grants; L.C.O. and A.L.D.B. performed the genotyping and statistical analysis; L.C.O. and G.A.C. drafted the manuscript. All authors contributed to and approved the final version of this manuscript based on critical revision.

\section{References}

1 Ingram CJ, Mulcare CA, Itan Y, Thomas MG, Swallow DM. Lactose digestion and the evolutionary genetics of lactase persistence. Hum Genet. 2009 Jan;124(6):579-91.

2 Swallow DM. Genetics of lactase persistence and lactose intolerance. Annu Rev Genet. 2003;37(1):197-219.

3 Itan Y, Jones BL, Ingram CJ, Swallow DM, Thomas MG. A worldwide correlation of lactase persistence phenotype and genotypes. BMC Evol Biol. 2010 Feb;10(1):36.

4 Enattah NS, Sahi T, Savilahti E, Terwilliger JD, Peltonen L, Järvelä I. Identification of a variant associated with adult-type hypolactasia. Nat Genet. 2002 Feb;30(2):233-7.

5 Lewinsky RH, Jensen TG, Møller J, Stensballe A, Olsen J, Troelsen JT. T-13910 DNA variant associated with lactase persistence interacts with Oct- 1 and stimulates lactase promoter activity in vitro. Hum Mol Genet. 2005 Dec; 14(24):3945-53.
6 Leseva MN, Grand RJ, Klett H, Boerries M, Busch $\mathrm{H}$, Binder AM, et al. Differences in DNA Methylation and Functional Expression in Lactase Persistent and Non-Persistent Individuals. Sci Rep. 2018 Apr;8(1):5649.

7 Bartel DP. MicroRNAs: target recognition and regulatory functions. Cell. 2009 Jan; 136(2):215-33.

8 Kim DH, Saetrom P, Snøve O Jr, Rossi JJ. MicroRNA-directed transcriptional gene silencing in mammalian cells. Proc Natl Acad Sci USA. 2008 Oct;105(42):16230-5.

9 Mishra PJ, Mishra PJ, Banerjee D, Bertino JR. miRSNPs or miR-polymorphisms, new players in microRNA mediated regulation of the cell: introducing microRNA pharmacogenomics. Cell Cycle. 2008 Apr;7(7):853-8.
10 Bhattacharya A, Ziebarth JD, Cui Y. PolymiRTS Database 3.0: linking polymorphisms in microRNAs and their target sites with human diseases and biological pathways. Nucleic Acids Res. 2014;42(D1):86-91.

11 Arola H. Diagnosis of hypolactasia and lactose malabsorption. Scand J Gastroenterol Suppl. 1994;202:26-35.

12 Boschmann SE, Boldt AB, de Souza IR, PetzlErler ML, Messias-Reason IJ. The Frequency of the LCT*-13910>CT Polymorphism Associated with Lactase Persistence Diverges among Euro-Descendant Groups from Brazil. Med Princ Pract. 2016;25(1):18-20.

13 Sudmant PH, Rausch T, Gardner EJ, Handsaker RE, Abyzov A, Huddleston J, et al.; 1000 Genomes Project Consortium. An integrated map of structural variation in 2,504 human genomes. Nature. 2015 Oct;526(7571):75-81. 